\title{
Thyrotoxic Periodic Paralysis (TPP) in a 28-Year- Old Sudanese Man Started on Prednisone
}

\author{
Joshua J. Tessier, DO, Stacey K. Neu, MD, and Kristin K. Horning, PharmD
}

Thyrotoxic periodic paralysis is a rare complication associated with hyperthyroidism. It is most common in the Asian population. We describe a case of a 28 -year-old Sudanese man who presented with ascending lower extremity paralysis after treatment of hyperthyroidism with prednisone and methimazole. This case report reviews the literature looking at pathophysiology, causes, and treatment recommendations for this rare complication of hyperthyroidism. (J Am Board Fam Med 2010;23:551-554.)

Keywords: Thyrotoxic Periodic Paralysis, TPP, Prednisone, Sudanese, Case Report

Hyperthyroidism is a relatively common condition in the United States, affecting $1.2 \%$ of the population. ${ }^{1}$ The treatment of hyperthyroidism consists of $\beta$-blockers for symptom control and thyroid ablation, which is accomplished either radioactively with radioiodine (I131) or chemically using methimazole or propylthiouracil. ${ }^{2}$ Thyrotoxic periodic paralysis (TPP), a rare sequelae of hyperthyroidism, is more common among Asian men and is thought to be associated with high carbohydrate loads. ${ }^{3-6}$ This report examines a case of TPP believed to be precipitated by the initiation of chemical thyroid ablation plus prednisone in a young Sudanese man. We will discuss the potential relationship between medications and TPP and explore a theory regarding the physiologic processes that lead to hypokalemia in hyperthyroidism.

\section{Case Report}

A 28-year-old man of Sudanese descent presented to the emergency department at approximately 7:30 AM complaining of weakness. He had awak-

This article was externally peer reviewed.

Submitted 18 September 2009; revised 8 February 2010; accepted 15 February 2010.

From the Iowa Lutheran Family Medicine Residency, Iowa Lutheran Hospital, Des Moines (JJT, KKH, SKN); and University of Iowa College of Pharmacy (KKH), Iowa City.

Funding: none.

Conflict of interest: none declared.

Corresponding author: Joshua J. Tessier, DO, Iowa Lutheran Family Medicine Residency, East Des Moines Family Care Center, 840 East University Avenue, Des Moines, IA 50316 (E-mail: tessiejj@ihs.org). ened approximately 3 hours earlier with weakness primarily in his lower extremities and again 2 hours later to find that the profound weakness had now spread to his upper extremities. He reported no other neurological symptoms either before or during this episode. About 6 weeks earlier he had been seen by his primary physician for an unintentional 20-pound weight loss, palpitations, and insomnia. Laboratory abnormalities included impaired fasting glucose and elevated thyroid function tests. He was diagnosed with hyperthyroidism, started on atenolol, and was referred to an endocrinologist. Treatment with methimazole, propranolol, and prednisone subsequently was initiated.

At the time of his emergency department visit the patient was otherwise healthy and denied tobacco, alcohol, or drug use. He had emigrated from Sudan 1 year earlier. Pertinent family history included one brother with hyperthyroidism and no known cases of periodic paralysis. A review of systems revealed occasional fevers, night-time coughing, and palpitations during the last several weeks.

During physical examination the patient appeared slightly anxious with no altered mental status. He was afebrile with a pulse of 96 beats per minute, respirations of 16 breaths per minute, pulse oximetry of $100 \%$ on room air, and blood pressure $152 / 60 \mathrm{~mm} \mathrm{Hg}$. His neck was supple without thyromegaly or lymphadenopathy. Neurologic examination revealed 3 of 4 bilateral reflexes in the patellar, biceps, and achilles regions and muscle strength of 3 of 5 on the left and 4 of 5 on the right side in both the upper and lower extremities. No 
Table 1. Abnormal Laboratory Values During Hospitalization

\begin{tabular}{|c|c|c|c|c|c|}
\hline Test (Reference) & Day 0, 0800 Hours & Day 0,1400 Hours & Day 0,1800 Hours & Day 1 & Day 2 \\
\hline Potassium (3.6-5.1 mmol/L) & 2.0 & 3.4 & 4.0 & 3.6 & 4.4 \\
\hline Glucose $(70-100 \mathrm{mg} / \mathrm{dL})$ & 128 & & & 95 & 93 \\
\hline $\mathrm{FT}_{3}(2.3-4.2 \mathrm{pg} / \mathrm{mL})$ & 9.0 & & & & \\
\hline $\mathrm{T}_{4}(4.5-10.9 \mathrm{mcg} / \mathrm{dL})$ & 19.4 & & & & \\
\hline TSH (0.35-5.5 mcIU/mL) & 0.013 & & & & \\
\hline Urine potassium $(\mathrm{mmol} / \mathrm{L})$ & & 23 & & & \\
\hline
\end{tabular}

$\mathrm{FT}_{3}$, free triiodothyronine; $\mathrm{T}_{4}$, thyroxine total; $\mathrm{TSH}$, thyroid-stimulating hormone.

sensory or cranial nerve deficits were noted. His heart rate was regular and his heart rhythm was without murmurs; initial electrocardiogram revealed sinus rhythm and probable left ventricular hypertrophy. Although serial cardiac enzymes were mildly elevated, no evidence of ischemia was found on further workup. Computed tomography scan of the head was negative. Abnormal laboratory studies are listed in Table 1.

At admission, the differential diagnosis included primary hyperaldosteronism, hypokalemic periodic paralysis, thyrotoxic periodic paralysis, other periodic paralyzes, and myopathies. After physical examination and an evaluation of his laboratory values the patient was diagnosed with TPP. The dose of propranolol was increased, prednisone was discontinued, and potassium supplementation was given. Within a few hours the patient showed marked improvement and remained without residual neurological symptoms. On hospital day 2 he was discharged on a treatment regimen of methimazole, propranolol, and potassium. He was readmitted 8 months later with a potassium level of 1.5 and similar symptoms. The patient admitted that he did not adhere to the drug therapy. After the patient improved using the same medication regimen, it was presumed that noncompliance resulted in recurrence of the TPP. The patient was subsequently discharged and referred back to the endocrinologist.

\section{Discussion}

Hyperthyroidism is associated with a number of muscular disorders, including acute and chronic thyrotoxic myopathies, exophthalmic ophthalmoplegia, myasthenia gravis, and periodic paralysis. ${ }^{4}$ TPP is almost exclusively seen in men and tends to affect younger adults, with $85 \%$ of patients developing symptoms between 20 and 39 years of age. ${ }^{3,4}$
A review of several case reports showed that individuals who consume a high carbohydrate intake followed by prolonged rest are the most likely to be affected by this condition. ${ }^{3,4,6,7}$ Although TPP is the rarest of hyperthyroidism's complications, it is more common in certain patient populations, with a reported incidence of $2 \%$ in Japanese patients with hyperthyroidism. ${ }^{4,5}$ The incidence is likely to increase given our multicultural population, excessive obesity, and the predominance of insulin resistance.

Our patient, a 28-year-old Sudanese man with impaired fasting glucose, awoke with progressive weakness 6 weeks after being diagnosed with hyperthyroidism. A pattern of sequential motor involvement beginning in the lower limbs and ascending to the upper limbs is typical for TPP. Attacks range in severity from mild weakness to flaccid paralysis, with recovery occurring first in those muscles affected last. Upper motor involvement is uncommon and mental faculties are never impaired. Respiratory muscle involvement, though rare, usually proves fatal. ${ }^{4}$

Acute management of TPP consists of potassium chloride supplementation, careful observation, and close monitoring of serial potassium concentrations. ${ }^{4}$ A usual dose of potassium is $130 \mathrm{mEq}$ divided over the first 24 hours, with subsequent doses based on serial potassium concentrations. Intravenous potassium is required if the patient shows signs of cardiac dysrhythmia or respiratory distress or is unable to take oral medications. ${ }^{4}$ According to current guidelines from the American Association of Clinical Endocrinologists, hyperthyroidism should be treated with I131, antithyroid medications, or surgery. ${ }^{2}$ Though antithyroid agents may be used to induce remission, the success of this intervention is variable and relapses are frequent. After discussion with the endocrinologists, it seems 
that methimazole was chosen because of patient refusal and the possibility of long-term medication requirements with I131 ablation in a young patient. Chemical ablation has primarily shown benefit in elderly, cardiac, pregnant, and pediatric patients. ${ }^{2}$

Although hypokalemia is common among patients with TPP, it can be challenging to understand this relationship. It is known that hyperthyroidism affects plasma membrane permeability to sodium and potassium by increasing $\mathrm{Na}+-\mathrm{K}+$-adenosinetriphosphatase (ATPase) activity in skeletal muscle, liver, and kidney cells. ${ }^{6,7}$ In addition, $\beta$-adrenergic sensitivity is increased, which augments $\mathrm{Na}+-\mathrm{K}+$-ATPase activity. The $\mathrm{Na}+-\mathrm{K}+$-ATPase pump is also activated by insulin, which may explain the connection of the paralytic attacks to the consumption of heavy carbohydrate meals. ${ }^{6}$ Some animal studies have indicated that $\mathrm{Na}+-\mathrm{K}+-$ ATPase activity is stimulated by androgens and is inhibited by estrogens and progesterones, which may account for the predominance of the disorder among men. ${ }^{6}$

Given this theory, nonselective $\beta$-blockers such as propranolol have been shown to markedly improve thyrotoxic symptoms and provide relief of paralytic episodes by inhibiting the intracellular shift of potassium induced by epinephrine. ${ }^{8}$ Selective $\beta$-blockers, including metoprolol, do not protect patients from paralytic attacks, which is consistent with the specific role of the $\beta_{2}$ receptor in mediating the catecholamine-induced increase in $\mathrm{Na}+-\mathrm{K}+$-ATPase activity in skeletal muscle. ${ }^{6} \mathrm{De}-$ spite potential benefits, $\beta$-blockers, particularly nonselective agents, are known to have a slight detrimental effect on carbohydrate metabolism and other metabolic effects. ${ }^{9}$ Our patient received oral propranolol before and during his admission and the dose was actually increased in an attempt to control his tachycardia and hypertension. No worsening of the hypokalemia was noted.

The role of glucocorticoids in the treatment of hyperthyroidism has been studied in a variety of settings. Prednisone has been shown to induce and sustain remission of hyperthyroidism in one small study. ${ }^{10}$ In the setting of thyroid storm, glucocorticoids are used in patients with adrenal insufficiency and hypotension. ${ }^{11}$ In addition, they can serve as adjunctive therapy to reduce triiodothyronine (T3) levels by inhibiting the peripheral conversion of thyroxine (T4) to triiodothyronine. Despite these potential benefits, the role of glu- cocorticoids has typically been limited to the prevention of worsening ocular symptoms in patients with known Graves ophthalmopathy. ${ }^{2}$

Although glucocorticoids have been used to treat hyperthyroidism, they may also produce detrimental effects, including the development of TPP. A literature review revealed at least 2 cases of TPP induced by methylprednisolone and 2 cases caused by a single dose of prednisone. ${ }^{6,12-14}$ In contrast, our patient received prednisone for 7 days before being diagnosed with TPP. In addition to inhibiting insulin secretion from the $\beta$-cells of the pancreas, glucocorticoids exhibit metabolic effects on carbohydrate, protein, and fat metabolism, thereby affecting peripheral glucose uptake. As previously discussed, one possible trigger for an attack of TPP is the ingestion of a high carbohydrate load. ${ }^{3}$ Because of the detrimental effects on insulin sensitivity, glucocorticoids can mimic this physiologic process and induce these attacks.

\section{Conclusion}

TPP is a relatively rare phenomenon in the United States. Despite the potential insulin resistance induced by nonselective $\beta$-blockers, these agents have been shown to abort attacks of TPP and are still recommended to control symptoms until definitive treatment can be initiated. Glucocorticoids should be reserved for treatment of ophthalmoplegia; several case reports of TPP after their administration have been published. ${ }^{2,6,12-14}$ Ablative therapy with I131 could have prevented the significant and potentially fatal reoccurrence of TPP in this patient because it would have resulted in earlier definitive treatment and not require long-term adherence to medication. Early recognition of TPP and potassium replacement remain the standard of care in preventing morbidity and mortality from this disorder.

\section{References}

1. Hollowell JG, Staehling NW, Flanders WD, et al. Serum TSH, T(4), and thyroid antibodies in the United States population (1988 to 1994): National Health and Nutrition Examination Survey (NHANES III). J Clin Endocrinol Metab 2002;87: 489-99.

2. Baskin HJ, Cobin RH, Duick DS, et al. American Association of Clinical Endocrinologists medical guidelines for clinical practice for the evaluation and treatment of hyperthyroidism and hypothyroidism. Endocr Pract 2002;8:457-69. 
3. El-Hennawy AS, Nesa M, Mahmood AK. Thyrotoxic hypokalemic periodic paralysis triggered by high carbohydrate diet. Am J Ther 2007;14:499501.

4. Tinker TD, Vannatta JB. Thyrotoxic hypokalemic periodic paralysis: report of four cases and review of the literature (1). J Okla State Med Assoc 1987;80: 11-5.

5. Feely J. Potassium shift in thyrotoxic periodic paralysis. Postgrad Med J 1981;57:238-9.

6. Ober KP. Thyrotoxic periodic paralysis in the United States. Report of 7 cases and review of the literature. Medicine (Baltimore) 1992;71:109-20.

7. Layzer RB. Periodic paralysis and the sodium-potassium pump. Ann Neurol 1982;11:547-52.

8. Shayne P, Hart A. Thyrotoxic periodic paralysis terminated by intravenous propranolol. Ann Emerg Med 1994;24:736-40.

9. Dehoff-Cooper RM, Pacanowski MA, Pepine CJ. Cardiovascular therapies and associated glucose homeostasis: implications across the dysglycemia continuum. J Am Coll Cardiol 2009;53:S28-34.

10. Werner SC, Platman SR. Remission of hyperthyroidism (Graves' disease) and altered pattern of serum-thyroxine binding induced by prednisone. Lancet 1965;2:751-5.

11. Nayak B, Burman K. Thyrotoxicosis and thyroid storm. Endocrinol Metab Clin N Am 2006;35:66386.

12. Liu Z, Braverman LE, Malabanan A. Thyrotoxic periodic paralysis in a hispanic man after the administration of prednisone. Endocr Pract 2006;12:42731.

13. Wongraoprasert S, Buranasupkajorn P, Sridama V, Snabboon T. Thyrotoxic periodic paralysis induced by pulse methylprednisolone. Intern Med 2007;46: 1431-3.

14. Miyashita Y, Monden T, Yamamoto K, et al. Ventricular fibrillation due to severe hypokalemia induced by steroid treatment in a patient with thyrotoxic periodic paralysis. Intern Med 2006;45:11-3. 